\title{
Hypoferraemia during the early inflammatory response is dependent on tumour necrosis factor activity in a murine model of protracted peritonitis
}

\author{
THOMAS E.O. SCHUBERT ${ }^{1,5}$, ANJA K. BOSSERHOFF ${ }^{1}$, CAROLE PEYSSONAUX $^{3}$, BERND ECHTENACHER ${ }^{2}$, \\ MITCHELL KNUTSON ${ }^{4}$, FERDINAND HOFSTÄDTER ${ }^{1}$ and DANIELA N. MÄNNEL ${ }^{2}$ \\ Departments of ${ }^{1}$ Pathology and ${ }^{2}$ Immunology, University of Regensburg, Regensburg, Germany; \\ ${ }^{3}$ Department of Endocrinology, Metabolism and Cancer, INSERM U567, Faculty Cochin, Paris, France; \\ ${ }^{4}$ Food Science and Human Nutrition Department, University of Florida, Gainesville, Florida, USA; \\ ${ }^{5}$ Institute of Applied Pathology, Speyer, Germany
}

Received March 1, 2012; Accepted June 19, 2012

DOI: $10.3892 / \mathrm{mmr} .2012 .1004$

\begin{abstract}
On the grounds of clinical, in vitro and in vivo studies, tumour necrosis factor (TNF) is considered to be one of the inflammatory cytokines that contributes to to the generation of hypoferraemia and anaemia of inflammation (AI). We used a recently described murine model for AI and hypoferraemia, based on sublethal caecal ligation and puncture (CLP) with ensuing protracted peritonitis, to investigate the contribution of TNF to the generation of hypoferraemia. During the early inflammatory response to CLP, a marked decrease in serum iron concentration occurs within $8 \mathrm{~h}$. To determine whether TNF contributes to the generation of hypoferraemia at this time point, we studied TNF-deficient mice and wild-type mice that underwent CLP. The serum iron concentration was decreased in wild-type mice whereas TNF-deficient mice maintained normal serum iron levels following CLP. Hypoferraemia in wild-type mice was accompanied by the downregulation of ferroportin 1 (Fp1) in macrophages. In the macrophages of TNF-deficient mice, Fp1 was not downregulated following CLP. The initial expression of hepcidin was detectable at the mRNA level but not at the protein level by immunohistochemistry in wild-type and TNF-deficient mice. Therefore, hepcidin does not appear to be involved in the regulation of early hypoferraemia. TNF appears to regulate the expression of Fp1 by transcriptional control. Our results demonstrate that TNF mediates hypoferraemia during the early inflammatory response by regulating the expression of $\mathrm{Fpl}$ in macrophages.
\end{abstract}

Correspondence to: Dr Thomas Schubert, Institute of Applied Pathology, St.-Guido-Stifts-Platz 5, D-67346 Speyer, Germany

E-mail: dr.t.schubert@googlemail.com

Key words: hypoferraemia, blood, TNF, mouse, septic peritonitis

\section{Introduction}

Hypoferraemia is part of the inflammatory response (1). It is considered to be a defence mechanism of the host that prevents pathogenic organisms from gaining access to iron (2). Tumour necrosis factor (TNF) has been viewed as being one of the inflammatory cytokines that contributes considerably to the generation of hypoferraemia and anaemia of inflammation (AI). TNF has been shown to affect iron metabolism in murine models, in humans and in vitro. The experimental administration of TNF reportedly induced hypoferraemia (3) and anaemia with low serum iron and preserved iron stores in mice (4). A combination of interferon- $\gamma($ IFN- $\gamma$ ) and TNF was found to induce hypoferraemia, decrease transferrin and soluble transferrin receptor levels and increase ferritin levels in the sera of cancer patients treated with these cytokines by isolated limb perfusion (5). Iron release from macrophages was reported to be inhibited by TNF in vitro (6). Previous findings demonstrated that TNF regulates key molecules engaged in iron import and export in the small bowel independently of hepcidin $(3,7)$. TNF stimulation was found to upregulate the cellular iron import protein divalent metal transporter 1 (DMT1) and reduce the iron exporter ferroportin 1 (Fp1) in a human monocytic cell line (8), resulting in increased iron sequestration.

The reported findings demonstrate that TNF, by regulating several molecules crucial to iron absorption and iron recycling, has a considerable impact on iron metabolism. In this study, we investigated whether TNF contributes to the generation of hypoferraemia during the early inflammatory response in a murine model of protracted peritonitis in vivo. For this purpose, we studied TNF-deficient and wild-type mice undergoing caecal ligation and puncture (CLP).

\section{Materials and methods}

Mice. Female or male C57/BL6 mice (25-30 g) were bred at the animal facility of the University of Regensburg, Germany. TNF-deficient mice were generated by Körner et al (9), 
raised at the University of Erlangen, Germany, and provided by $\mathrm{H}$. Körner. The animals were kept under standard conditions at the animal facility of the University of Regensburg. Experiments were performed according to federal guidelines for animal experimentation. Animals in the control and treated groups were age- and gender-matched.

CLP. The mice were anaesthetised by i.p. injection of $75 \mathrm{mg} /$ kg Ketanest ${ }^{\circledR}$ (Parke, Davis \& Co., Munich, Germany) and 16 mg/kg Rompun ${ }^{\circledR}$ (Bayer AG, Leverkusen, Germany). The caecum was exteriorised and its distal end was ligated and punctured to achieve a sublethal CLP as described in a previous study (10).

Serum iron measurement and histology. The serum iron concentration was measured using the ADVIA ${ }^{\mathrm{TM}} 1650$ chemistry system (Bayer Diagnostics, Fernwald, Germany). To obtain serum, groups of mice $(n=5)$ were anaesthetised and blood was drawn $8 \mathrm{~h}$ after CLP. The mice were subsequently sacrificed by cervical dislocation and the spleen, liver and small bowel were either harvested for histological analysis or snap frozen in liquid nitrogen. For histology, mouse tissue was fixed in $4 \%$ buffered formalin overnight. Formalin-fixed tissue was dehydrated and embedded in paraffin. Paraffin sections of 3-5 $\mu \mathrm{m}$ were mounted on SuperFrost Plus slides, heated for $20 \mathrm{~min}$ at $72^{\circ} \mathrm{C}$, deparaffinised and rehydrated. Following antigen retrieval in a citrate buffer $(\mathrm{pH} 7.3)$ with microwave irradiation for $30 \mathrm{~min}$ at $240 \mathrm{~W}$, the slides were rinsed and endogenous peroxidase activity was blocked with methanolic peroxide. The slides were rinsed again and primary monoclonal antibodies against hemoxygenase 1 (Abcam, Cambridge, UK), NRAMP (Alpha Diagnostic International, San Antonio, TX, USA) or ferritin (Abcam), respectively, were applied in a dilution of 1:100. The slides were incubated using a Ventana machine (Ventana Medical Systems basic DAB detection kit; Ventana Medical Systems Inc., Tucson, AZ, USA). The streptavidin-biotin-peroxidase method was used for visualisation. Immunohistochemical stainings for hepcidin (Alpha Diagnostic International) and Fp1 (provided by M. Knutson) were applied in a dilution of 1:200 following antigen retrieval in a citrate buffer. To evaluate iron stores, the sections were stained by Perls' acid ferrocyanide reaction.

Isolation of RNA. Total cellular RNA was isolated using the RNeasy Mini kit including an RNase-free DNase set according to the manufacturer's instructions (Qiagen, Hilden, Germany). RNA was quantified using a fluorescence microplate reader and by following the instructions of the RiboGreen ${ }^{\circledR}$ RNA quantitation reagent and kit (Mobitec, Göttingen, Germany).

Quantification of $m R N A$ expression by real-time PCR. One-tenth of the RNA recovered from RNeasy spin columns (Qiagen) was used for reverse transcription PCR. cDNA fragments of hepcidin and Fp1 were amplified using the primers designed by Laftah et al (3). Reverse transcription was performed in a $20 \mu \mathrm{l}$ reaction mix of $5 \mu \mathrm{l}$ RNA, $4 \mu \mathrm{l}$ 5X SuperScript ${ }^{\mathrm{TM}}$ buffer (Invitrogen, Carlsbad, CA, USA), $2 \mu \mathrm{l}$ DTT $(0.1 \mathrm{M}), 1 \mu \mathrm{l}$ antisense dN6 primer $(1 \mu \mathrm{g} / \mu \mathrm{l}), 1 \mu \mathrm{l}$ dNTPs $(10 \mathrm{mM})$ and $1 \mu \mathrm{l}$ reverse transcriptase (SuperScript ${ }^{\mathrm{TM}}$, Invitrogen). The mixture was incubated at $46^{\circ} \mathrm{C}$ for $45 \mathrm{~min}$ followed by $10 \mathrm{~min}$ at $70^{\circ} \mathrm{C}$ and RNase A digestion at $37^{\circ} \mathrm{C}$ for $30 \mathrm{~min}$. To precisely quantify the expression of mRNA, the real-time PCR LightCycler ${ }^{\circledR}$ system (Roche Diagnostics, Mannheim, Germany) was used. For PCR, 1-3 $\mu$ l cDNA preparation, $2.4 \mu \mathrm{l} 25 \mathrm{mM} \mathrm{MgCl} \mathrm{M}_{2}, 0.5 \mu \mathrm{M}$ of forward and reverse primer and $2 \mu \mathrm{l}$ of SYBR Green LightCycler ${ }^{\circledR}$ Mix in a total of $20 \mu \mathrm{l}$ were applied. The following PCR programme was performed: $60 \mathrm{sec}$ at $95^{\circ} \mathrm{C}$ (initial denaturation); $20^{\circ} \mathrm{C} / \mathrm{sec}$ temperature transition rate up to $95^{\circ} \mathrm{C}$ for $15 \mathrm{sec}, 10 \mathrm{sec}$ at $58^{\circ} \mathrm{C}$, $22 \mathrm{sec}$ at $72^{\circ} \mathrm{C}$ and $10 \mathrm{sec}$ at $82^{\circ} \mathrm{C}$ (acquisition mode single), repeated 40 times (amplification). The $\mathrm{MgCl}_{2}$ concentration and annealing temperature were optimised for each primer set. The PCR was evaluated by melting curve analysis following the manufacturer's instructions and checking the PCR products on $1.8 \%$ agarose gels. Each quantitative PCR was performed at least in duplicate for two sets of RNA preparations.

ELISA assay. The sera of 4 animals per time point were pooled and maintained at $-20^{\circ} \mathrm{C}$ until analysis. TNF concentrations were tested using a sandwich ELISA assay (R\&D Systems, Wiesbaden, Germany). Absorption was measured at $405 \mathrm{~nm}$ and evaluated using SoftMax ${ }^{\circledR}$ software.

Statistical analysis. P-values were determined using the Student's t-test. $\mathrm{P}<0.05$ was considered to indicate a statistically significant result.

\section{Results}

The serum concentration of TNF peaks $8 \mathrm{~h}$ after CLP. The serum concentration of TNF in wild-type mice was measured over a period of $24 \mathrm{~h}$ after CLP. The TNF concentration increased rapidly within the first few hours and peaked $8 \mathrm{~h}$ after CLP. The TNF concentration subsequently decreased to almost baseline levels (Fig. 1).

Hypoferraemia does not develop in TNF-deficient mice $8 \mathrm{~h}$ after CLP. To test the role of TNF in the development of hypoferraemia, TNF-deficient mice were used to study the serum iron concentration following CLP. Wild-type mice showed a significant decrease in serum iron concentration $8 \mathrm{~h}$ after CLP (Fig. 2). The serum iron concentration of CLP-treated TNF-deficient animals did not differ significantly from the serum iron concentrations of the untreated control and untreated TNF-deficient animals.

Hypoferraemia is accompanied by the downregulation of Fpl in wild-type mice. Immunofluorescent staining of hepatic and splenic tissue revealed that the iron exporter Fp1 was downregulated in Kupffer cells and splenic macrophages in wild-type mice $8 \mathrm{~h}$ after CLP, whereas in enterocytes of the duodenum, the membrane staining pattern for Fp1 was unaffected (Fig. 3). In agreement with this finding, the mRNA level of Fp1 was decreased in mice following CLP compared with untreated animals (Fig. 4A). These changes were accompanied by an increase in cytoplasmic ferritin expression in Kupffer cells and macrophages after CLP as revealed by the immunohistochemical analysis (data not shown). Immunohistochemical analysis for DMT1 and hemoxygenase 1 did not reveal any differences between the treated and 


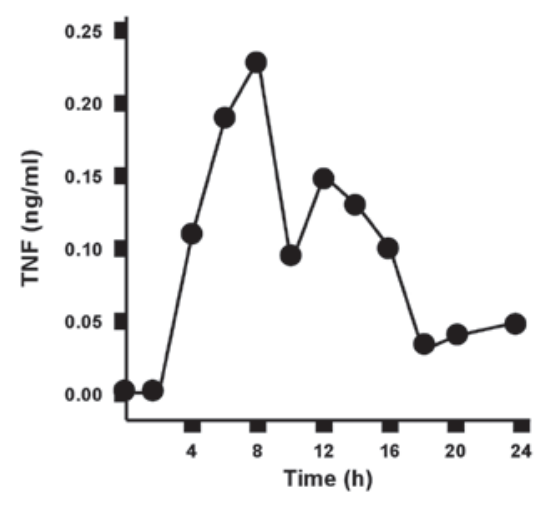

Figure 1. TNF serum concentrations markedly increased within the first $8 \mathrm{~h}$ after CLP. TNF serum concentrations in the wild-type mice after CLP were determined at the indicated time points by ELISA. Pooled serum from 4 animals was analysed per time point. TNF, tumour necrosis factor; CLP, caecal ligation and puncture.

untreated animals or between the wild-type and TNF-deficient mice (data not shown).

Fpl is not downregulated in the absence of TNF-activity following CLP. In TNF-deficient mice, immunofluorescent staining for Fpl after CLP exhibited membrane patterns similar to those of untreated mice (Fig. 3). Consequently, no changes in Fp1 mRNA expression were observed. Fp1 mRNA levels were equally increased in the untreated TNF-deficient mice and in the TNF-deficient mice after CLP compared with the wild-type mice (Fig. 4A).

No differences were found in the expression of Fp1 mRNA in the liver between the treated and untreated or wild-type and TNF-deficient mice (Fig. 4B).

Hepatic hepcidin expression is not increased $8 \mathrm{~h}$ after CLP in either wild-type or TNF-deficient mice. The splenic and hepatic hepcidin expression was determined in untreated wild-type and TNF-deficient mice $8 \mathrm{~h}$ after CLP by quantitative RT-PCR. An initial increase in the splenic hepcidin expression was observed in wild-type and TNF-deficient mice following CLP (Fig. 5A). The hepcidin mRNA levels were not increased $6 \mathrm{~h}$ after CLP (data not shown). Hepatic hepcidin expression was equally low in all groups $8 \mathrm{~h}$ after CLP, indicating that hepatic hepcidin had not increased (Fig. 5B). Immunohistochemically stained sections of the liver revealed that hepcidin peptide was not detectable in hepatocytes, Kupffer cells or the spleen (data not shown).

\section{Discussion}

Various effects of TNF on iron metabolism have been described in the literature. TNF, as a mediator of inflammation, is thought to affect iron metabolism, similar to IL-6 and other cytokines, in order to deprive pathogens of access to iron. However, the precise mechanism by which TNF impacts iron metabolism and the phase of inflammation in which this occurs are unknown.

Experimental administration of TNF to animals results in a blockade of iron recycling, iron absorption and hypoferraemia. High concentrations of TNF achieved by the administration of

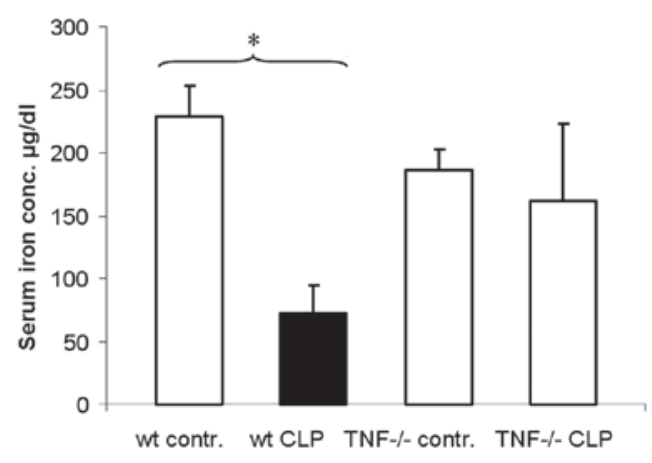

Figure 2. Hypoferraemia develops in wild-type but not TNF-deficient mice $8 \mathrm{~h}$ after CLP. Wild-type mice developed marked hypoferraemia $8 \mathrm{~h}$ after CLP compared with the untreated control mice $(\mathrm{p}<0.00001)$. The serum iron concentration of TNF-deficient mice after CLP did not decrease compared with the untreated wild-type and TNF-deficient mice. Results are given as mean $( \pm \mathrm{SD}, \mathrm{n}=5)$. TNF, tumour necrosis factor; CLP, caecal ligation and puncture; wt, wild-type. ${ }^{*} \mathrm{P}<0.05$ compared to wt control mice.

TNF-secreting tumour cells were found to induce anaemia, with a low serum iron concentration, and preserved iron stores in nude mice (4). The induction of hypoferraemia using low doses of TNF in mice has also been described by other authors $(6,11)$. Laftah et al demonstrated that the administration of TNF causes hypoferraemia associated with reduced intestinal iron absorption in mice (3). The concentrations used in this study equal the serum concentrations of TNF that we measured after CLP (Fig. 1). After CLP, we observed a rapid increase in TNF serum levels, peaking $8 \mathrm{~h}$ after CLP. At the same time point, marked hypoferraemia was observed in wild-type mice. To test whether hypoferraemia was mediated by TNF, we studied TNF-deficient mice following CLP. The data demonstrated that TNF-deficient mice did not develop hypoferraemia during the early inflammatory response. During CLP-induced hypoferraemia in wild-type mice, the expression levels of the iron importer DMT1 and hemoxygenase 1 were unchanged compared with those of the untreated animals. The membrane expression of the iron exporter Fp1 in splenic macrophages and Kupffer cells was reduced in wild-type mice following CLP whereas in the TNF-deficient mice, the membrane staining pattern of the macrophages was maintained. Fp1 membrane expression was not reduced in the duodenum of either the wild-type or TNF-deficient mice. These findings indicate that hypoferraemia is achieved by a TNF-induced downregulation of Fp1, the only known iron exporter protein in macrophages.

It has previously been reported that hepcidin, an iron regulatory peptide, regulates the degradation of Fp1 (12). During inflammatory conditions, hepcidin is produced in the liver and secreted into the blood. It binds to Fp1 located in the cell membranes of macrophages and enterocytes. Subsequently, Fp1 is internalised and degraded (12). The decrease of Fp1 in macrophages and enterocytes results in reduced iron efflux and the blockade of iron recycling as well as iron resorption (13). Macrophages and polymorphonuclear leukocytes constitute other sources of hepcidin (14). The endogenous expression of hepcidin in macrophages is mediated by TLR- 4 and causes the internalisation of Fp1 in an autocrine manner (14). Hepcidin expression in wild-type and TNF-deficient mice after CLP was studied to determine whether the TNF-induced downregulation 


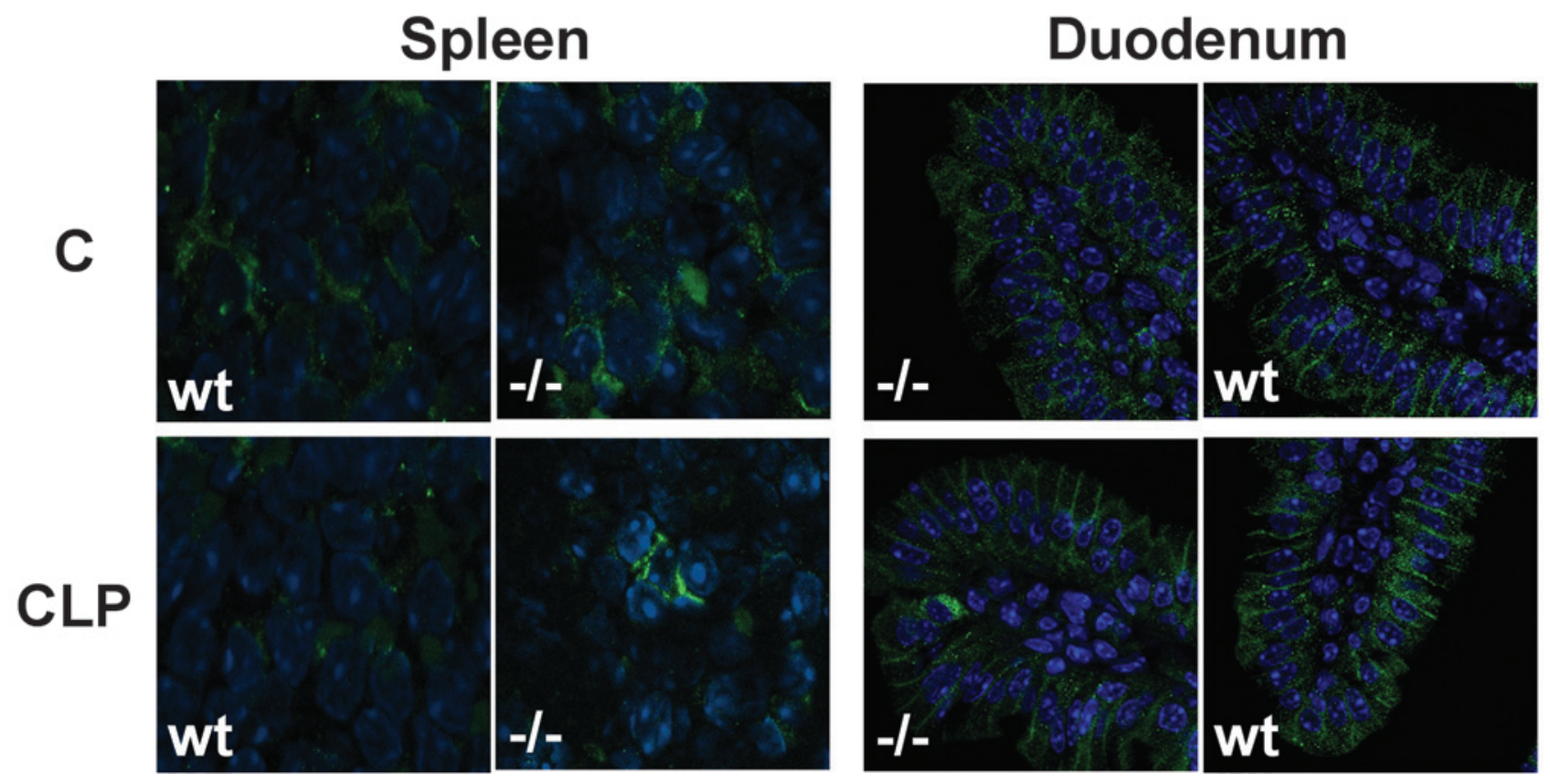

Figure 3. In the absence of TNF activity, Fp1 membrane expression is not reduced during the early inflammatory response following CLP. Sections of splenic tissue and duodenum (original magnification, $\mathrm{x} 40$ ) from the untreated control mice (C wt) and untreated TNF-deficient mice (C TNF-/-) with marked membrane staining of macrophages or enterocytes for Fp1. Following CLP, wild-type mice (CLP wt) showed a reduction of membrane staining of the splenic macrophages but not duodenal enterocytes. In TNF-deficient mice after CLP (CLP TNF-/-), the staining intensity for Fpl of splenic macrophages in the red pulp was not decreased. TNF, tumour necrosis factor; Fp1, ferroportin 1; CLP, caecal ligation and puncture; wt, wild-type.

A

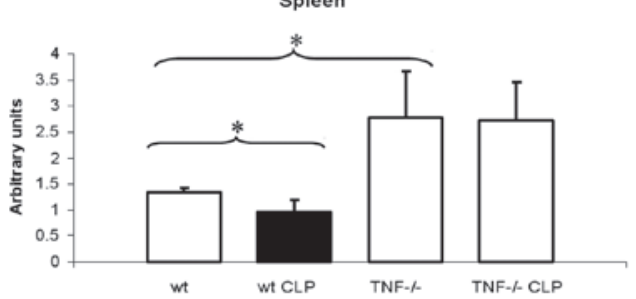

B

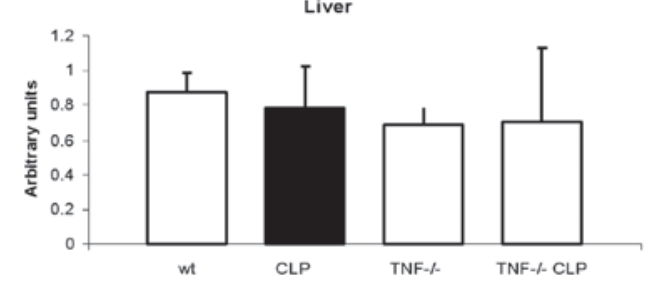

Figure 4. Expression of Fp1 mRNA is increased in the spleens of TNF-deficient mice and is not downregulated by acute inflammation in the absence of TNF activity. (A) Expression of Fp1 was determined in RNA isolated from the splenic tissue of the untreated and CLP-treated wild-type and TNF-deficient mice by quantitative RT-PCR. Untreated TNF-deficient mice and TNF-deficient mice undergoing CLP exhibited an increased level of Fp1 mRNA compared with wild-type mice ( $\mathrm{p}<0.05$ and $\mathrm{p}<0.05$, respectively). (B) Expression of Fp1 in the liver is not regulated by the early inflammatory response following CLP. The relative expression of $\mathrm{Fp} 1$ in the liver tissue of untreated and CLP-treated wild-type and TNF-deficient mice was measured by quantitative RT-PCR. Fp1, ferroportin 1; TNF, tumour necrosis factor; CLP, caecal ligation and puncture; wt, wild-type.

of Fpl is mediated by hepcidin. It appears unlikely that systemically available hepcidin causes the downregulation of Fpl since the hepatic expression of hepcidin did not increase after CLP. Furthermore, the internalisation of Fp1 was not observed in
A

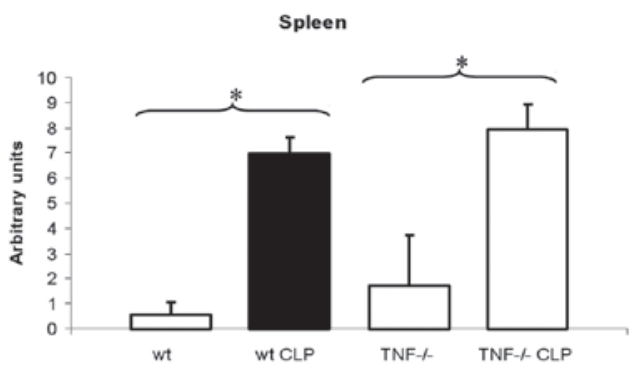

B

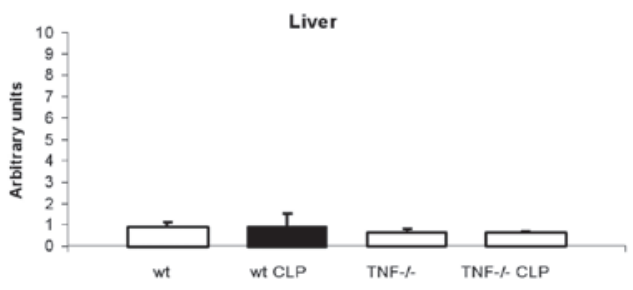

Figure 5. Expression of hepcidin mRNA is equally increased in the spleens of wild-type and TNF-deficient mice and is not upregulated in the liver $8 \mathrm{~h}$ after CLP. (A) Hepcidin mRNA was increased following CLP in wild-type and TNF-deficient mice ( $\mathrm{p}<0.001$ and $\mathrm{p}<0.01$, respectively). The expression of hepcidin was determined in RNA isolated from the splenic tissue of the untreated and CLP-treated wild-type and TNF-deficient mice by quantitative RT-PCR. (B) No difference was found in the hepatic hepcidin mRNA expression following CLP in wild-type and TNF-deficient mice compared with the untreated mice. A relative expression of hepcidin in liver tissue was measured by quantitative RT-PCR. TNF, tumour necrosis factor; CLP, caecal ligation and puncture; wt, wild-type.

duodenal enterocytes, indicating that the autocrine production of hepcidin may be the cause of hypoferraemia rather than a systemic hepcidin effect. The expression of hepcidin in splenic macrophages and Kupffer cells $8 \mathrm{~h}$ after CLP was detectable at 
the mRNA level but not at the protein level, negating the possibility that hepcidin is involved in mediating the downregulation of Fp1 by TNF. No difference was observed in hepcidin expression between the wild-type and TNF-deficient mice. TNF has marked hepcidin-independent effects on iron metabolism. Laftah et al reported that the application of TNF suppresses rather than stimulates hepcidin expression in the spleens and livers of CD1 mice and that TNF induces iron sequestration in the spleen and inhibits duodenal iron transfer independently of hepcidin (3). The same authors described a direct effect of TNF on the iron import and export of enterocytes, which was associated with a decrease in DMT1 and Fp1 expression (7). The rapid action of TNF on serum iron levels within $3 \mathrm{~h}$ after application reported by Laftah et al (3) apparently mediates hypoferraemia in the early inflammatory response before hepcidin production in the liver increases (Fig. 4). Moreover, for other models of inflammation it has been reported that hepcidin expression in the liver is not increased at this time point (15), indicating that it is unlikely that systemically available hepcidin is a mediator of hypoferraemia during the early inflammatory response. This is in line with our data, indicating that the TNF-induced immediate hypoferraemia following CLP is not mediated by hepcidin and that the downregulation of Fp1 must be achieved by a different mechanism.

In untreated and CLP-treated TNF-deficient mice, an enhanced Fp1 expression was observed at the mRNA and protein levels compared with wild-type mice, indicating that TNF regulates the Fp1 expression by transcriptional control. In wild-type mice, a decrease of Fp1 mRNA was observed after CLP. Downregulation of Fp1 by TNF was previously reported in human endothelial cells (16). LPS, a potent stimulator of TNF production, also suppresses Fp1 expression in macrophages, cultured splenic cells and in the mouse spleen $(8,17,18)$. The enhanced expression of Fp1 in untreated mice in the absence of TNF suggests that TNF regulates the transcription of Fp1 not only during inflammation, but also under physiological conditions.

Previously, we investigated the contribution of TNF to the different aspects of AI in vivo in the CLP model (19). Our results demonstrated that AI may develop during protracted inflammation in the absence of TNF two weeks after CLP. The role of TNF in the mediation of the chronic phase of AI is therefore questionable. Since TNF does not induce hepcidin production (3), it is unlikely that TNF is a mediator of hypoferraemia in the later inflammatory phase which is typically regulated by IL- 6 and hepcidin (20). This explains the reason for hypoferraemia and AI developing in the absence of TNF during the chronic phase of inflammation. However, in the early inflammatory response TNF appears to exert its well-described effects on iron metabolism and is necessary for the induction of hypoferraemia prior to the onset of hepcidin production.

In summary, our findings suggest that TNF is a mediator of hypoferraemia during the early inflammatory response by regulating the expression of Fp1 in macrophages independently of hepcidin.

\section{Acknowledgements}

The technical support of Simone Kaufmann is gratefully acknowledged.

\section{References}

1. Weiss G: Modification of iron regulation by the inflammatory response (Review). Best Pract Res Clin Haematol 18: 183-201, 2005.

2. Schaible UE and Kaufmann SH: Iron and microbial infection. Nat Rev Microbiol 2: 946-953, 2004.

3. Laftah AH, Sharma N, Brookes MJ, McKie AT, Simpson RJ, Iqbal TH and Tselepis C: Tumour necrosis factor alpha causes hypoferraemia and reduced intestinal iron absorption in mice. Biochem J 397: 61-67, 2006.

4. Johnson RA, Waddelow TA, Caro J, Oliff A and Roodman GD: Chronic exposure to tumor necrosis factor in vivo preferentially inhibits erythropoiesis in nude mice. Blood 74: 130-138, 1989.

5. Feelders RA, Vreugdenhil G, Eggermont AM, KuiperKramer PA, van Eijk HG and Swaak AJ: Regulation of iron metabolism in the acute-phase response: interferon gamma and tumour necrosis factor alpha induce hypoferraemia, ferritin production and a decrease in circulating transferrin receptors in cancer patients. Eur J Clin Invest 28: 520-527, 1998.

6. Alvarez-Hernández X, Licéaga J, McKay IC and Brock JH: Induction of hypoferremia and modulation of macrophage iron metabolism by tumor necrosis factor. Lab Invest 61 : 319-322, 1989.

7. Sharma N, Laftah AH, Brookes MJ, Cooper B, Iqbal T and Tselepis C: A role for tumour necrosis factor alpha in human small bowel iron transport. Biochem J (Pt 2) 390: 437-446, 2005.

8. Ludwiczek S, Aigner E, Theurl I and Weiss G: Cytokinemediated regulation of iron transport in human monocytic cells. Blood 101: 4148-4154, 2003.

9. Körner H, Cook M, Riminton DS, Lemckert FA, Hoek RM, Ledermann B, Köntgen F, Fazekas de St Groth B and Sedgwick JD: Distinct roles for lymphotoxin-alpha and tumor necrosis factor in organogenesis and spatial organization of lymphoid tissue. Eur J Immunol 27: 2600-2609, 1997.

10. Schubert TE, Echtenacher B, Hofstädter F and Männel DN: Failure of interferon- $\gamma$ and tumor necrosis factor in mediating anemia of chronic disease in a mouse model of protracted septic peritonitis. Int J Mol Med 16: 753-758, 2005.

11. Tanaka T, Araki E, Nitta K and Tateno M: Recombinant human tumor necrosis factor depresses serum iron in mice. J Biol Response Mod 6: 484-488, 1987.

12. Nemeth E, Tuttle MS, Powelson J, Vaughn MB, Donovan A, Ward DM, Ganz T and Kaplan J: Hepcidin regulates cellular iron efflux by binding to ferroportin and inducing its internalization. Science 306: 2090-2093, 2004.

13. Rivera S, Nemeth E, Gabayan V, Lopez MA, Farshidi D and Ganz T: Synthetic hepcidin causes rapid dose-dependent hypoferremia and is concentrated in ferroportin-containing organs. Blood 106: 2196-2199, 2005.

14. Peyssonnaux C, Zinkernagel AS, Datta V, Lauth X, Johnson RS and Nizet V: TLR4-dependent hepcidin expression by myeloid cells in response to bacterial pathogens. Blood 107: 3727-3732, 2006.

15. Nicolas G, Chauvet C, Viatte L, Danan JL, Bigard X, Devaux I, Beaumont C, Kahn A and Vaulont S: The gene encoding the iron regulatory peptide hepcidin is regulated by anemia, hypoxia, and inflammation. J Clin Invest 110: 1037-1044, 2002.

16. Nanami M, Ookawara T, Otaki Y, Ito K, Moriguchi R, Miyagawa K, Hasuike Y, Izumi M, Eguchi H, Suzuki K and Nakanishi T: Tumor necrosis factor-alpha-induced iron sequestration and oxidative stress in human endothelial cells. Arterioscler Thromb Vasc Biol 25: 2495-2501, 2005.

17. Liu XB, Nguyen NB, Marquess KD, Yang F and Haile DJ: Regulation of hepcidin and ferroportin expression by lipopolysaccharide in splenic macrophages. Blood Cells Mol Dis 35: 47-56, 2005.

18. Yang F, Liu XB, Quinones M, Melby PC, Ghio A and Haile DJ: Regulation of reticuloendothelial iron transporter MTP1 (Slc11a3) by inflammation. J Biol Chem 277: 39786-39791, 2002.

19. Schubert T, Echtenacher B, Hofstädter F and Männel DN: TNF-independent development of transient anemia of chronic disease in a mouse model of protracted septic peritonitis. Lab Invest 83: 1743-1750, 2003.

20. Ganz T and Nemeth E: Iron sequestration and anemia of inflammation. Semin Hematol 46: 387-393, 2009. 